\title{
HSCT in Elderly Patients
}

\author{
Rafael F. Duarte and Isabel Sánchez-Ortega
}

\subsection{Introduction}

The hematological malignancies which are the most common indications for auto- and alloHSCT (e.g., AML/MDS, NHL, MM, and others) are diagnosed at a median age greater than 65 years old. Thus, if classical chronological age exclusion criteria were followed, a majority of patients with these malignancies would not be offered a HSCT, despite it being their treatment of choice and in many cases their only curative option (Sureda et al. 2015). While elderly patients are more likely to face toxic effects from HSCT, this risk must be considered and balanced against the poor outcome of transplant candidates with these malignancies who do not proceed to HSCT.

\subsection{HSCT Activity in Elderly Patients}

Auto- and allo-HSCT annual activity continues to steadily increase in Europe and worldwide with no signs of saturation (Gratwohl et al. 2015). Specifically, in elderly patients, HSCT activity at

R. F. Duarte $(\bowtie)$

Hospital Universitario Puerta de Hierro

Majadahonda, Madrid, Spain

e-mail: rduarte.work@gmail.com

I. Sánchez-Ortega

Institut Català d'Oncologia Hospitalet,

Barcelona, Spain
EBMT centers has increased markedly in the past two decades. Auto-HSCT activity in patients $\geq 65$ years old increased from $3.4 \%$ (443 out of 13,163 autologous HSCT) in 2000 to $9.8 \%$ (2444 out of 23,883 auto-HSCT) in 2014 (SánchezOrtega et al. 2016). Allo-HSCT activity in patients $\geq 65$ years old increased from $<1 \%$ (37 out of 6413 allo-HSCT) in 2000 to $6.7 \%$ (1057 out of 16,765 allogeneic HSCT) in 2014 (Basak et al. 2016). In the USA, over $50 \%$ of auto-HSCT for lymphomas and MM were performed in patients over 60 years old and $12 \%$ in patients $\geq 70$ years old in 2015 (D'Souza and Zhu 2016). The number of patients aged $\geq 60$ undergoing allo-HSCT doubled in 20072013 compared to 2000-2006 (D'Souza and Zhu 2016), and US allografts for patients $\geq 70$ years rose tenfold over the past decade, with AML as the leading indication (Muffly et al. 2016).

Improvements in supportive care, HSC mobilization, and the use of RTC and RIC regimens have contributed to the increase in HSCT activity overall and, in particular, to the increase of HSCT activity rates in elderly patients. With sustained improvement in these areas, and as the population ages, these numbers will only continue to increase.

\subsection{HSCT Outcomes in Elderly Patients}

Compared to younger adults, elderly patients may have higher overall rates of transplant failure. Potential comorbidities, impaired health, 
and performance status could lead to higher transplant-related morbidity and mortality. In addition, malignancies in elderly patients often have more adverse disease features (e.g., higher-risk cytogenetics and molecular patterns in AML/MDS patients) and may have been treated less aggressively prior to HSCT, which may potentially also increase the risk of disease relapse.

Historically, HSCT outcome analysis in elderly patients has been limited by the fact that these patients are underrepresented in clinical trials and the majority of data come from relatively small series and subgroup analyses of small subsets of elderly patients in larger disease-specific studies including adults of all ages. More recently, HSCT outcomes of elderly patients are being analyzed specifically and have reported feasibility and safety of autologous HSCT in MM patients $>65$ years (Winn et al. 2015), in selected populations of elderly patients with R/R DLBCL (Chihara et al. 2014), and in $R / R$ HL in patients $\geq 60$ years of age (Martínez et al. 2017). Prospective studies addressing the value of allogeneic HSCT compared to non-transplant approaches are limited and generally restricted to patients $<65$ years old. Interestingly, several large series in AML/ MDS patients reported that NRM and OS were negatively affected by KPS $<80-90 \%$ but not by chronological age (Heidenreich et al. 2017; McClune et al. 2010). Despite significantly poorer outcomes in older patients, additional trials have also not shown a significant impact of advanced age on major outcomes including NRM (Sorror et al. 2011; Chevallier et al. 2012).

The largest experience reported to date on auto- and allo-HSCT outcomes in elderly patients comes from two EBMT studies including a total of 21,390 auto-HSCT and 6046 alloHSCT in patients $\geq 65$ years old between 2000 and 2014 (Basak et al. 2016; Sánchez-Ortega et al. 2016). Patient numbers and key HSCT outcomes overall and by age group are presented in Table 68.1.

These studies confirm the feasibility of autoand allo-HSCT in elderly patients, with accept-
Table 68.1 HSCT outcomes in elderly patients: EBMT experience $^{\mathrm{a}}$

\begin{tabular}{l|l|l|l} 
Type of HSCT & $\begin{array}{l}\text { All cases } \\
\geq 65 \text { years }\end{array}$ & $\begin{array}{l}\text { Group I } \\
65-69 \text { years }\end{array}$ & $\begin{array}{l}\text { Group II } \\
\geq 70 \text { years }\end{array}$ \\
\hline Autologous, $n$ & 21,390 & 17,531 & 3859 \\
- NRM year 1 & $4.9 \%$ & $4.6 \%$ & $5.9 \%$ \\
- OS year 1 & $87 \%$ & $88 \%$ & $83 \%$ \\
- OS year 3 & $67 \%$ & $69 \%$ & $61 \%$ \\
Allogeneic, $n$ & 6046 & 4914 & 1132 \\
- NRM year 1 & $27 \%$ & $26 \%$ & $29 \%$ \\
- OS year 1 & $57 \%$ & $57 \%$ & $53 \%$ \\
- OS year 3 & $39 \%$ & $40 \%$ & $35 \%$
\end{tabular}

$n$ number of cases

aBasak et al. (2016) and Sánchez-Ortega et al. (2016)

able NRM and OS at 1 and 3 years. Multivariate analyses in both studies showed that performance status (i.e., Karnofsky score) had a more significant independent impact on patient outcomes than chronological age. Thus, these data in a large cohort of elderly patients strongly suggest that age per se should not be an exclusion criterion to consider HSCT in this population. Undoubtedly, this is presumably a highly selected fraction of elderly patients considered for auto- and alloHSCT. Nevertheless, this further endorses the need to assess comorbidity and frailty beyond age in older HSCT candidates to improve outcomes further.

\subsection{Assessment of Elderly Candidates for HSCT}

In addition to the elements already discussed in Chap. 11 for younger patients, the evaluation and counseling of elderly patients as candidates for auto- and allo-HSCT require the evaluation of additional health domains of interest in patients of advanced age. The following tables address general principles and considerations for evaluation and counseling of these patients, discuss the issue of patient frailty beyond age and comorbidities, and describe the key elements of a multidimensional geriatric assessment in this population. 


\subsubsection{General Principles and Considerations for Elderly HSCT Candidates}

- HSCT decision should not be driven by chronological age but by a broader multidimensional assessment including fitness, comorbidities, physiologic reserve, and frailty.

- Elderly patients require information and counseling in plain language regarding the HSCT process, donor sources, specific protocol, timeline, risks, benefits, and outcomes.

- They also need information regarding patients' quality of life outcomes, caregivers, and psychosocial needs, for which social workers and other support staff will be needed.

- A multidisciplinary individualized assessment is required to appropriately address the multidimensional nature of the evaluation of elderly patients.

- Fit older transplant candidates should follow the same indications for auto- and allo-HSCT as younger adults.

- In the case of allo-HSCT, particular consideration to RIC and NMA regimens is essential, and donor selection must take into account the age of the donor, as donor older age may associate with impaired outcomes.

- Outcome analysis in elderly patients may require the use of clinically relevant composite endpoints that, beyond survival, incorporate quality of life, good overall mental and physical condition, and freedom from severe complications.

\subsubsection{Frailty in Elderly HSCT Candidates}

- Frailty is a term used to describe a multidimensional syndrome of loss of physiologic reserves (energy, physical ability, cognition, health) that leads to vulnerability.

- The ability to measure frailty in elderly patients is useful clinically.

- Although it appears to be a valid construct to assess elderly patients, how exactly to define it remains unclear. There is a large abundance of possible scales to measure frailty, which likely reflects uncertainty about the term and its components.

- A. Hedge et al. have recently reported on frailty as the missing piece of pre-HSCT assessment (Hegde and Murthy 2018). Data shows that the prevalence of frailty prior to HSCT in patients $\geq 50$ years old is higher than in the general geriatric population at around $25 \%$. Importantly, age has no effect on prevalence of frailty.

- Frailty is associated with poorer OS even after adjusting for age and HCT-CI and may be associated as well with an increased incidence of disease relapse (Muffly et al. 2014, Hegde and Murthy 2018).

\subsubsection{Geriatric Assessment for Elderly HSCT Candidates}

\subsubsection{General Concept}

- The geriatric assessment is a multidimensional, multidisciplinary assessment designed to evaluate an older person's functional ability, physical health, cognition, mental health, and socioenvironmental circumstances (Artz 2016).

- The goal of geriatric assessment in this context would be to capture vulnerability preHSCT to help deciding on patient suitability for the procedure, as well as to individualize post-HSCT support strategies to prevent complications and reduce transplant-associated morbidity and mortality (Artz 2016).

\subsubsection{Elements Involved in Elderly HSCT Candidates}

- Ensure appropriate performance status (Karnofsky score $\geq 80$ ).

- Rule out significant comorbidities by the HCT-CI (Sorror et al. 2005), as their prevalence increases with age.

- Assess the modified EBMT (Armand et al. 2014) and the revised PAM scores (Au et al. 2015), as global prognostic models that incorporate both NRM and disease factors. 
- Measure functional status by self-reported function and performance-based testing (ability to perform tasks necessary to live independently in the community [i.e., shopping, food preparation, housekeeping, telephone, laundry, transportation and driving, manage finances and medication, number of times a patient can rise from the chair (i.e., timed up and go), gait speed, 6-min walk test, hand grip strength, or provocative cardiopulmonary testing], polypharmacy requirements).

- Cognitive function: if necessary, perform neuropsychological testing and/or consult geriatrics.

- Psychosocial evaluation (assessment of social support, availability of a caregiver, financial matters, psychological disturbances, etc.).

- Nutritional status and weight loss.

- Biomarkers to characterize physiologic age (serum C-reactive protein, ferritin, serum albumin, or protein biomarkers panels in development).

\subsubsection{Scales}

- No standard geriatric assessment scales have been validated for HSCT.

- Most scales available for geriatric assessment in cancer patients are complex and time-consuming, which limits its use in daily practice.

- The Geriatric Assessment in Hematology (GAH) scale is a brief, comprehensive geriatric assessment scale designed and validated for older patients diagnosed with hematological malignancies (MDS, AML, MM, and CLL) (Bonanad et al. 2015).

- The GAH scale includes 30 items grouped into 8 pre-defined dimensions (number of drugs, gait speed, mood, activities of daily living, subjective health status, nutrition, mental status, and comorbidities) and requires a relatively short period of time to be administered in routine clinical practice (10-12 min).

- Thus, the GAH scale could be an interesting tool to assess elderly patients with hematological malignancies who are being considered for transplantation. However, it still needs to be validated in the setting of HSCT.

\section{Key Points}

- HSCT activity in elderly patients has increased markedly in the past two decades and is predicted to continue to increase as the population ages, with a sustained improvement in HSCT methodology and care.

- Auto- and allo-HSCT in elderly patients is feasible and has acceptable outcomes.

- Age should not be an exclusion criterion per se to consider elderly patients for HSCT.

- Assessing comorbidity is essential in older HSCT candidates, but adjusting only for comorbidity may not identify frail patients vulnerable to adverse outcomes.

- Frailty is a multidimensional syndrome of loss of physiologic reserves (energy, physical ability, cognition, health) that leads to vulnerability, is higher in HSCT recipients than the general geriatric population, and associates with poorer HSCT outcome.

- Geriatric assessment is a multidimensional, multidisciplinary assessment designed to evaluate an older person's functional ability, physical health, cognition, mental health, and socioenvironmental circumstances.

- The goal of geriatric assessment in HSCT would be to capture vulnerability to pre-HSCT to help deciding on patient suitability for the procedure and to adapt post-HSCT support strategies to improve outcomes.

- Currently, there are no standard geriatric assessment scales validated for HSCT. The GAH scale has been described and validated in elderly patients with hematological malignancies, is relatively simple to apply in clinical practice, and may be a candidate scale for elderly HSCT candidates, validation pending. 


\section{References}

Armand P, Kim HT, Logan BR, et al. Validation and refinement of the disease risk index for allogeneic stem cell transplantation. Blood. 2014;123: 3664-71.

Artz AS. Biologic vs physiologic age in the transplant candidate. Hematology Am Soc Hematol Educ Program. 2016;2016:99-105.

$\mathrm{Au}$ BK, Gooley TA, Armand P, et al. Reevaluation of the pretransplant assessment of mortality score after allogeneic hematopoietic transplantation. Biol Blood Marrow Transplant. 2015;21:848-54.

Basak GW, Sánchez-Ortega I, Beohou E, et al. Allogeneic hematopoietic cell transplantation in elderly patients aged 65 and older: a retrospective analysis by the complications and quality of life working party of the EBMT. Blood. 2016;128:681.

Bonanad S, De la Rubia J, Gironella M, et al. Development and psychometric validation of a brief comprehensive health status assessment scale in older patients with hematological malignancies: the GAH scale. J Geriatr Oncol. 2015;6:353-61.

Chevallier P, Szydlo RM, Blaise D, et al. Reducedintensity conditioning before allogeneic hematopoietic stem cell transplantation in patients over 60 years: a report from the SFGM-TC. Biol Blood Marrow Transplant. 2012;18:289-94.

Chihara D, Izutsu K, Kondo E, et al. High-dose chemotherapy with autologous stem cell transplantation for elderly patients with relapsed/refractory diffuse large B cell lymphoma: a nationwide retrospective study. Biol Blood Marrow Transplant. 2014;20: 684-9.

D'Souza A, Zhu X. Current uses and outcomes of hematopoietic cell transplantation (HCT): CIBMTR summary slides. 2016. Available at http://www.cibmtr.org.

Gratwohl A, Pasquini MC, Aljurf M, et al. One million haematopoietic stem-cell transplants: a retrospective observational study. Lancet Haematol. 2015;2:e91-100.

Hegde A, Murthy HS. Frailty: the missing piece of the pre-hematopoietic cell transplantation assessment? Bone Marrow Transplant. 2018;53:3-10.

Heidenreich S, Ziagkos D, de Wreede LC, et al. Allogeneic stem cell transplantation for patients age $>/=70$ years with myelodysplastic syndrome: a retrospective Study of the MDS Subcommittee of the Chronic
Malignancies Working Party of the EBMT. Biol Blood Marrow Transplant. 2017;23:44-52.

Martínez C, Jorge AS, Pereira A, Hodgkin Lymphoma Subcommittee of Spanish Group of Lymphoma and Bone Marrow Transplantation (GELTAMO), et al. Comorbidities, not age, are predictive of survival after autologous hematopoietic cell transplantation for relapsed/refractory Hodgkin's lymphoma in patients older than 50 years. Ann Hematol. 2017;96:9-16.

McClune BL, Weisdorf DJ, Pedersen TL, et al. Effect of age on outcome of reduced-intensity hematopoietic cell transplantation for older patients with acute myeloid leukemia in first complete remission or with myelodysplastic syndrome. J Clin Oncol. 2010;28:1878-87.

Muffly LS, Kocherginsky M, Stock W, et al. Geriatric assessment to predict survival in older allogeneic hematopoietic cell transplantation recipients. Haematologica. 2014;99:1373-9.

Muffly L, Pasquini MC, Martens M, et al. Increasing use of allogeneic hematopoietic cell transplantation (HCT) in patients age 70 years and older: a CIBMTR study of trends and outcomes. Bone Marrow Transplant. 2016;22:S68-9.

Sánchez-Ortega I, Basak G, Beohou E, et al. Performance status drives the impact of age on the outcomes of autologous hematopoietic cell transplantation in elderly patients aged 65 and older: a retrospective analysis by the Complications and Quality of Life Working Party of the EBMT. Blood. 2016; 128:678.

Sorror ML, Maris MB, Storb R, et al. Hematopoietic cell transplantation (HCT)-specific comorbidity index: a new tool for risk assessment before allogeneic HCT. Blood. 2005;106:2912-9.

Sorror ML, Sandmaier BM, Storer BE, et al. Long-term outcomes among older patients following nonmyeloablative conditioning and allogeneic hematopoietic cell transplantation for advanced hematologic malignancies. JAMA. 2011;306:1874-83.

Sureda A, Bader P, Cesaro S, et al. Indications for alloand auto-SCT for haematological diseases, solid tumours and immune disorders: current practice in Europe, 2015. Bone Marrow Transplant. 2015;50: 1037-56.

Winn AN, Shah GL, Cohen JT, et al. The real world effectiveness of hematopoietic transplant among elderly individuals with multiple myeloma. J Natl Cancer Inst. 2015;107:1-6.

Open Access This chapter is licensed under the terms of the Creative Commons Attribution 4.0 International License (http://creativecommons.org/licenses/by/4.0/), which permits use, sharing, adaptation, distribution and reproduction in any medium or format, as long as you give appropriate credit to the original author(s) and the source, provide a link to the Creative Commons license and indicate if changes were made.

The images or other third party material in this chapter are included in the chapter's Creative Commons license, unless indicated otherwise in a credit line to the material. If material is not included in the chapter's Creative Commons license and your intended use is not permitted by statutory regulation or exceeds the permitted use, you will need to obtain permission directly from the copyright holder. 